\title{
A comprehensive study of spallation models for proton-induced spallation product yields utilized in transport calculation
}

\author{
Hiroki Iwamoto $^{1, *}$, Shin-ichiro Meigo ${ }^{1,}$, and Hiroki Matsuda ${ }^{1,}$ \\ ${ }^{1}$ J-PARC Center, Japan Atomic Energy Agency, 2-4, Shirakata, Tokai-mura, Naka-gun, Ibaraki 319-1195, Japan
}

\begin{abstract}
In order to understand the current status of the predictive performance of spallation models and issues towards further improvement, a comprehensive study of the proton-induced spallation product yields was conducted. In this study, four kinds of the latest spallation models (i.e. INCL++/ABLA07, INCL++/GEMINI++, INCL4.6/GEM, and CEM03.03), utilized in Monte Carlo particle transport calculation, are employed, and six pieces of cross section data measured at GSI, RIKEN, and J-PARC are used for comparison. As a result, the INCL++/ABLA07 calculations are in general agreement with the GSI and RIKEN experimental data, whereas some discrepancies are observed especially in the comparisons with the J-PARC experiments. Finally, several key issues inherent in each spallation model are described.
\end{abstract}

\section{Introduction}

In the neutronic and shielding design of high-energy accelerator facilities such as accelerator-driven systems (ADSs) and spallation neutron sources, spallation models implemented in Monte Carlo particle transport codes [1-4] provide us the information of spallation product yields, as well as energy spectra and angular distributions of secondary particles. Although an exhaustive benchmark analysis was conducted under the auspices of the International Atomic Energy Agency (IAEA) [5 ] and a great deal of effort has been devoted to improving the spallation models, discrepancies with experimental data, especially for the spallation product yields, still have been reported in recent analyses [6,7 ].

To understand the current status of the predictive performance of the spallation models and their issues towards further improvement, a comprehensive study for the proton-induced spallation product yields was conducted.

\section{Method}

In this study, the proton-induced nuclide production cross sections were calculated using different kinds of spallation models based on the intranuclear cascade (INC) plus deexcitation model (i.e., INCL++/ABLA07 [8,9], INCL++/GEMINI++ [10], INCL4.6/GEM [11,12], and CEM03.03 [13]), which are summarized in Table1. Here CEM03.03 contains the deexcitation model GEM developed by Furihata [12], in which some parameters were modified to account for fission cross sections; this model is referred to as GEM2 [14]. For the calculations by INCL++/ABLA07 and INCL++/GEMINI++, a stand-alone nuclear reaction simulation code, developed by Mancusi,

\footnotetext{
*e-mail: iwamoto.hiroki@jaea.go.jp
}

et al. [15], was employed. The INCL4.6/GEM and CEM03.03 calculations were performed by the use of nuclear reaction calculation options of the Particle and Heavy Ion Transport code System (PHITS) version 3.08 and the general-purpose Monte Carlo N-Particle code (MCNP) version 6.1 , respectively. The calculated cross sections were compared with experimental data measured at GSI [16-19], RIKEN [20], and J-PARC [21] as listedin Table 2, where the GSI data were taken from the EXchange FORmat (EXFOR) experimental nuclear reaction database [22].

Table 1. Spallation models used.

\begin{tabular}{lll}
\hline model name & INC & deexcitation \\
\hline INCL++/ABLA07 & INCL++ (ver.6.0) & ABLA07 \\
INCL++/GEMINI++ & INCL++ (ver.6.0) & GEMINI++ \\
INCL4.6/GEM & INCL (ver.4.6) & GEM \\
CEM03.03 & CEM03.03 & GEM2 \\
\hline
\end{tabular}

Table 2. Experimental data used for comparison.

\begin{tabular}{lrll}
\hline target & $E_{p}(\mathrm{MeV})$ & institute & reference no. \\
\hline${ }^{208} \mathrm{~Pb}$ & 1000 & GSI & {$[16]$} \\
${ }^{238} \mathrm{U}$ & 1000 & GSI & {$[17,18]$} \\
${ }^{136} \mathrm{Xe}$ & 1000 & GSI & {$[19]$} \\
${ }^{137} \mathrm{Cs}$ & 185 & RIKEN & {$[20]$} \\
${ }^{209} \mathrm{Bi}$ & 400 & J-PARC & {$[21]$} \\
${ }^{209} \mathrm{Bi}$ & 3000 & J-PARC & {$[21]$} \\
\hline
\end{tabular}

\section{Results and discussion}

\subsection{Comparison with GSI data for ${ }^{208} \mathrm{~Pb}$ and ${ }^{238} \mathrm{U}$}

Figures 1 and 2 illustrate the calculated mass number distributions of the spallation products for the $p(1 \mathrm{GeV})$ 


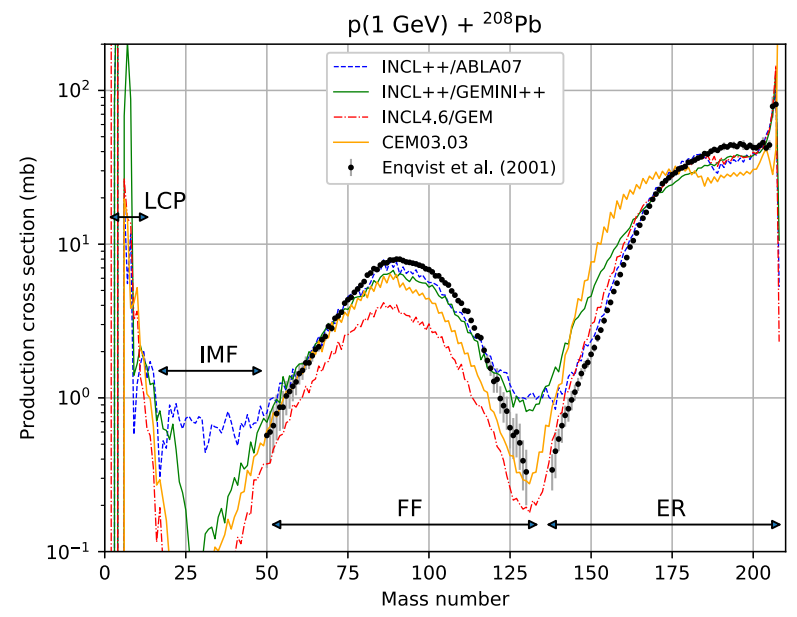

Figure 1. Comparison of mass number distribution between the GSI experiment and the model calculations for $p(1 \mathrm{GeV})+{ }^{208} \mathrm{~Pb}$ reaction.

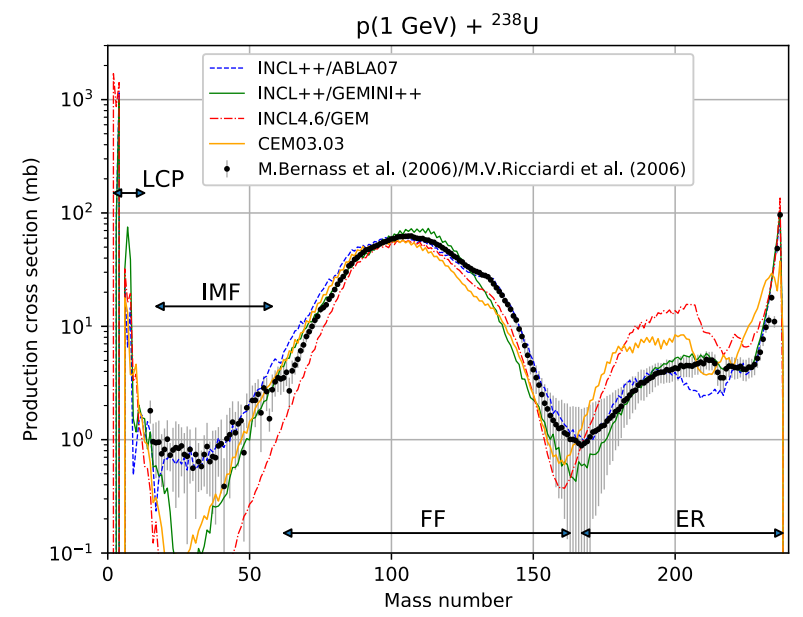

Figure 2. Same as Figure 1, but for $p(1 \mathrm{GeV})+{ }^{238} \mathrm{U}$ reaction.

$+{ }^{208} \mathrm{~Pb}$ and $p(1 \mathrm{GeV})+{ }^{238} \mathrm{U}$ reactions, respectively, together with experimental data measured at GSI with the inverse-kinematics technique. Here, we classify the spallation products into four groups according to their production mechanisms: light charged particle (LCP), intermediate mass fragment (IMF), fission fragment (FF), and evaporation residue (ER). A single Gaussian-like hump for mass numbers from 50 to 130 in Figure 1 and that for mass numbers from 60 to 150 in Figure 2 indicate FFs. Meanwhile, the spallation products at mass numbers higher than FFs indicate ERs. For both FFs and ERs, considerable discrepancies can be observed between the experimental data and the model calculations. For IMFs, no experimental data are found in EXFOR for the $p(1 \mathrm{GeV})$ $+{ }^{208} \mathrm{~Pb}$ reaction; however, Figure 2 suggests that IMFs are presumably produced from the spallation reactions, for which only ABLA07 appears to account for the IMF production.

To understand the discrepancies observed in Figures 1 and 2 in detail, we define an index of difference from
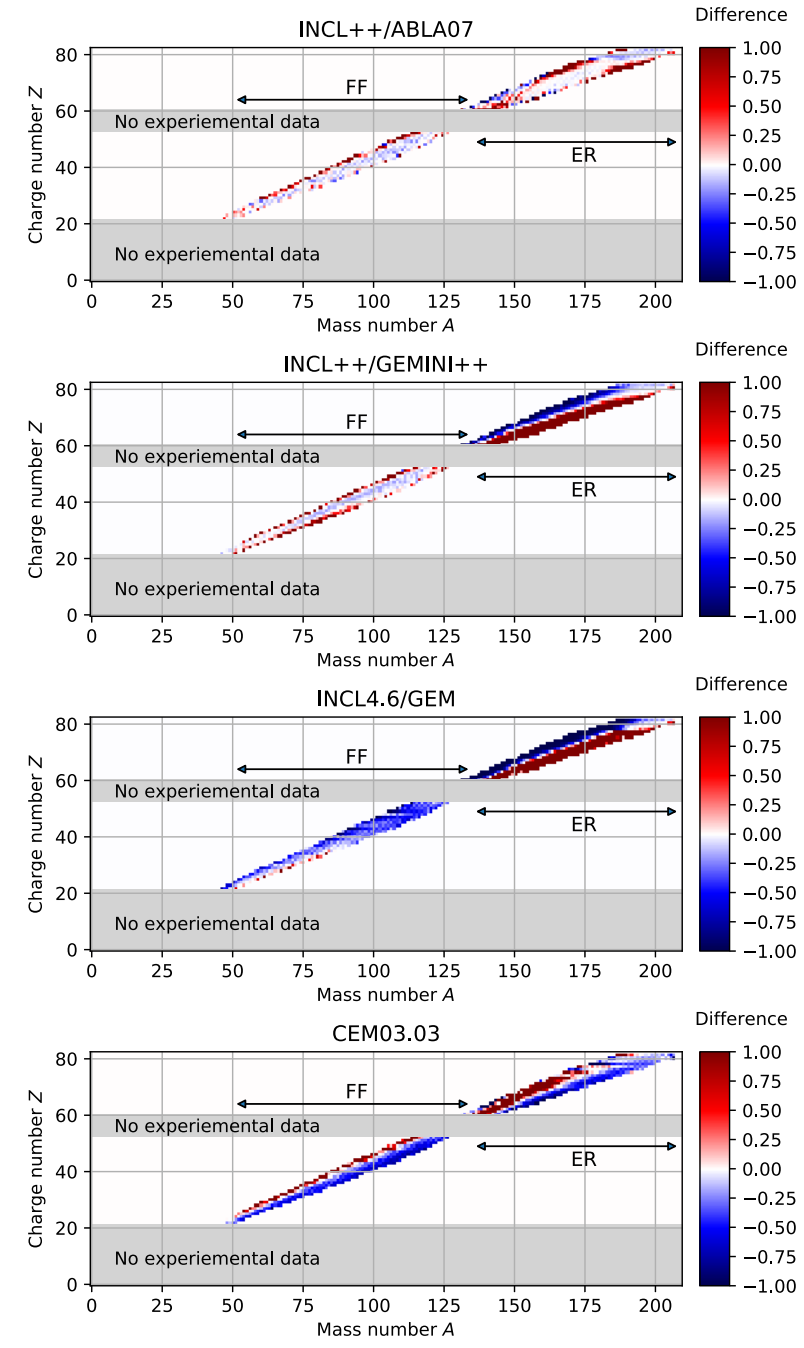

Figure 3. Two-dimensional $\eta$ distribution between the GSI experiment and model calculations for the $p(1 \mathrm{GeV})+$ ${ }^{208} \mathrm{~Pb}$ reaction (top panel: INCL++/ABLA07, upper panel: INCL++/GEMINI++, lower panel: INCL4.6/GEM, bottom panel: CEM03.03).

the experimental data $(\eta)$ for each $(A, Z)$ value:

$$
\begin{aligned}
\eta(A, Z) \equiv & \operatorname{sgn}\left(\sigma_{\text {calc }}(A, Z)-\sigma_{\text {expt }}(A, Z)\right) \\
& \cdot\left(\frac{\sigma_{\text {calc }}(A, Z)-\sigma_{\text {expt }}(A, Z)}{\sigma_{\text {expt }}(A, Z)}\right)^{2},
\end{aligned}
$$

where $A$ and $Z$ denote the mass and proton (or charge) numbers of the spallation products, respectively; $\sigma_{\text {calc }}$ and $\sigma_{\text {expt }}$ represent the calculated and experimental nuclide production cross sections, respectively.

Figure 3 displays the two-dimensional $\eta$ distribution between the GSI experimental data and the model calculations for the $p(1 \mathrm{GeV})+{ }^{208} \mathrm{~Pb}$ reaction, where the red and blue colors denote "overestimation" and "underestimation", respectively, whereas the white color indicates "agreement". It is seen from the top panel that INCL++/ABLA07 broadly reproduces the experimental data. In contrast, blue, white, and red colored stripes are observed in the ER region for the upper and lower panels, and the opposite stripe is seen in the bottom panel. 

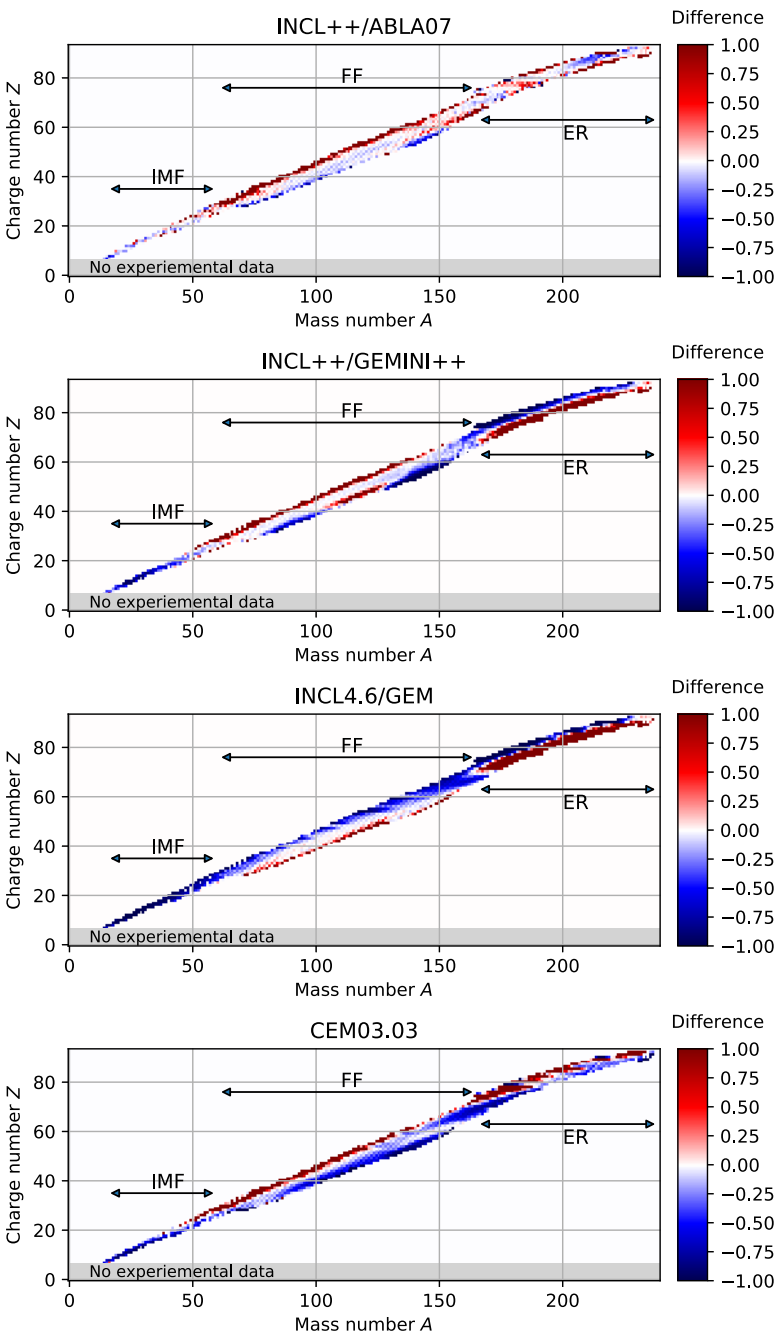

Figure 4. Same as Figure 3, but for $p(1 \mathrm{GeV})+{ }^{238} \mathrm{U}$ reaction.

This means that INCL++/GEMINI++ and INCL4.6/GEM estimate ERs as neutron rich for the same $Z$ number, whereas CEM03.03 estimates ERs as neutron poor for the same $Z$ number, implying that parametrizations in GEMINI++, GEM, and GEM2, would not match those of INCL++, INCL4.6, and CEM, respectively. Furthermore, as pointed out in Iwamoto et al. [6,23], it is seen in this figure that INCL4.6/GEM overall underestimates FFs.

Figure ?? shows the two-dimensional $\eta$ distribution between the GSI experimental data and the model calculations for the $p(1 \mathrm{GeV})+{ }^{238} \mathrm{U}$ reaction. The trends of $\eta$ distributions are similar to the case of the ${ }^{208} \mathrm{~Pb}$ target; that is, INCL++/ABLA07 shows overall in good agreement with the experimental data, and INCL++/GEMINI++ and INCL4.6/GEM estimate ERs as neutron rich for the same $Z$ number, whereas CEM03.03 estimates ERs as neutron poor for the same $Z$ number. As mentioned before, INCL++/GEMINI++, INCL4.6/GEM, and CEM03.03 underestimate IMFs, which would be attributed to the lack of the description of the IMF production in the deexcitation models.

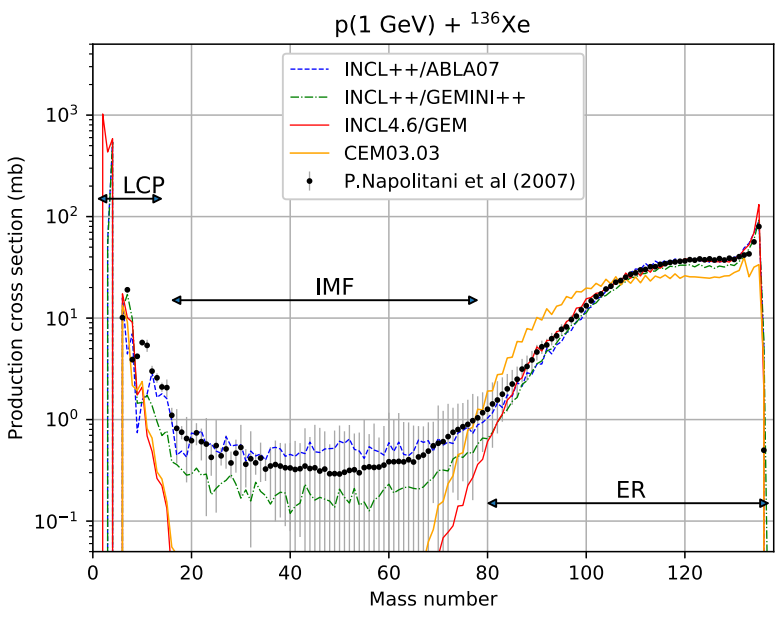

Figure 5. Comparison of mass number distribution between the GSI experiment and the model calculations for $p(1 \mathrm{GeV})+{ }^{136} \mathrm{Xe}$ reaction.
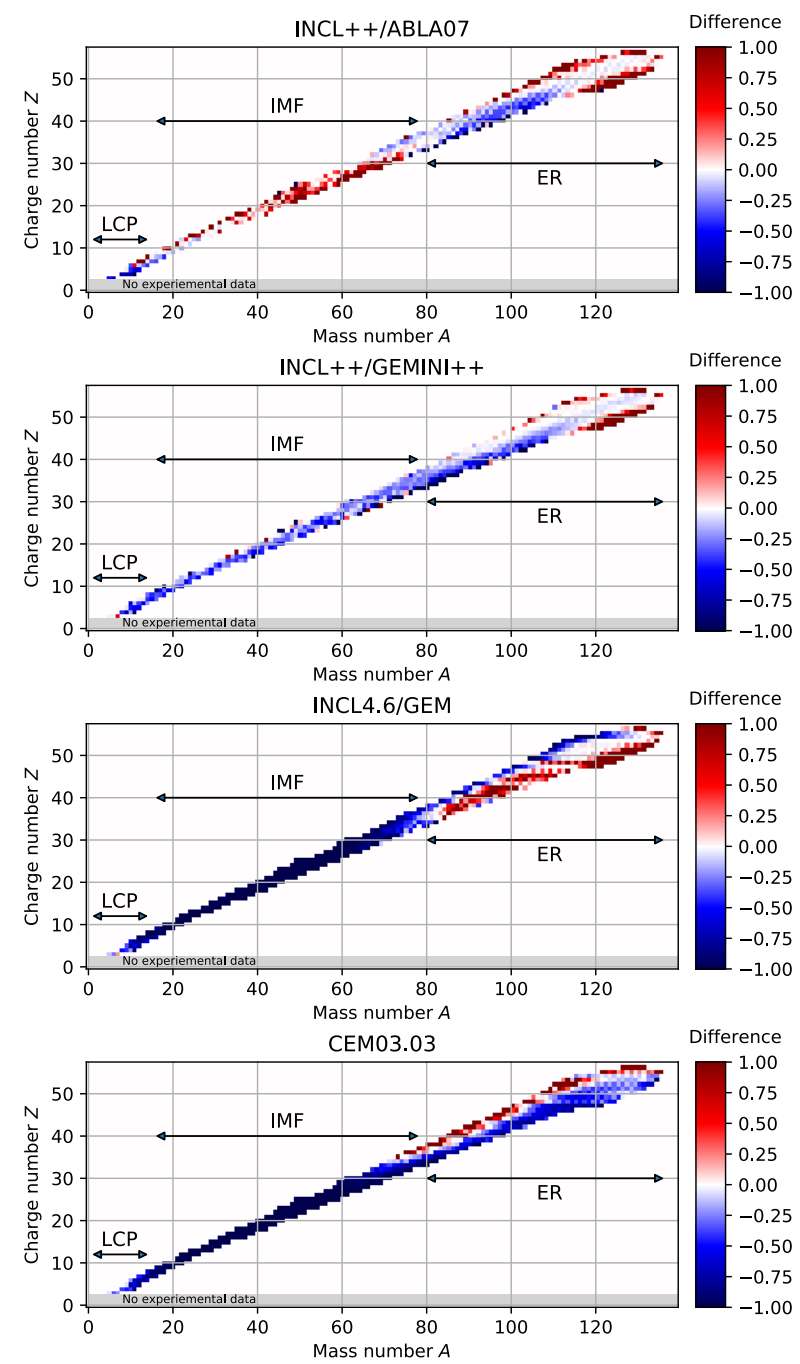

Figure 6. Same as Figure 3, but for $p(1 \mathrm{GeV})+{ }^{136} \mathrm{Xe}$ reaction.

\subsection{Comparison with GSI data for ${ }^{136} \mathrm{Xe}$ and RIKEN data for ${ }^{137} \mathrm{Cs}$}

Figures 5 and 6 show the mass number distributionsof the spallation products and the two-dimensional $\eta$ dis- 


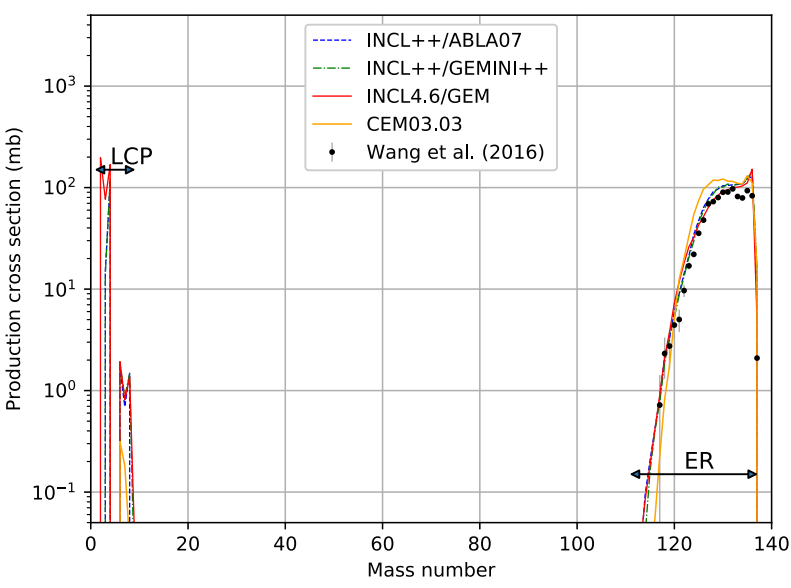

Figure 7. Comparison of mass number distribution between the RIKEN experiment and the model calculations for $p(185 \mathrm{MeV})$ $+{ }^{137}$ Cs reaction.
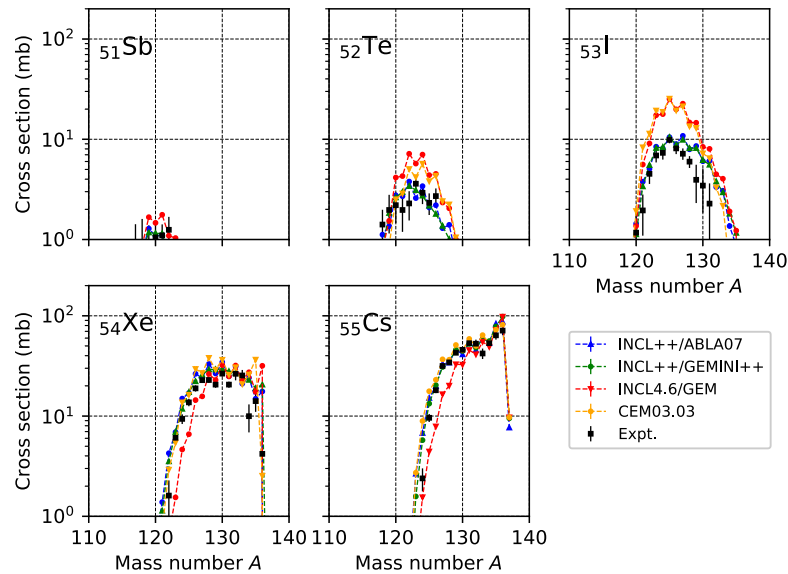

Figure 8. Comparison of mass number distribution for each produced isotope between the RIKEN experiment and the model calculations for $p(185 \mathrm{MeV})+{ }^{137} \mathrm{Cs}$ reaction.

tribution, respectively, for the $p(1 \mathrm{GeV})+{ }^{136} \mathrm{Xe}$ reaction. In this case, fission does not occur because of the light target mass. Although, at first sight, INCL++/ ABLA07, INCL++/GEMINI++, and INCL4.6/GEM reproduce the mass number distribution in the ER region, each model has their respective trends, and some of them are similar to the ${ }^{208} \mathrm{~Pb}$ and ${ }^{238} \mathrm{U}$ targets. The INCL++/ABLA07 overall reproduces the experimental IMF production cross sections within the experimental uncertainties, while underestimation is observed at mass numbers from 80 to 110 . Moreover, the INCL4.6/GEM estimates ERs as neutron rich for the same $Z$ number, whereas CEM03.03 estimates ERs as neutron poor for the same $Z$ number. In contrast to the ${ }^{208} \mathrm{~Pb}$ and ${ }^{238} \mathrm{U}$ target cases, the deexcitation model GEMINI++ reproduces the experimental IMF production cross sections for the $p(1 \mathrm{GeV})+{ }^{136} \mathrm{Xe}$ reaction within the experimental uncertainties.

Figure 7 illustrates the mass number distribution of the spallation products for the $p(185 \mathrm{MeV})+{ }^{137} \mathrm{Cs}$ reac-

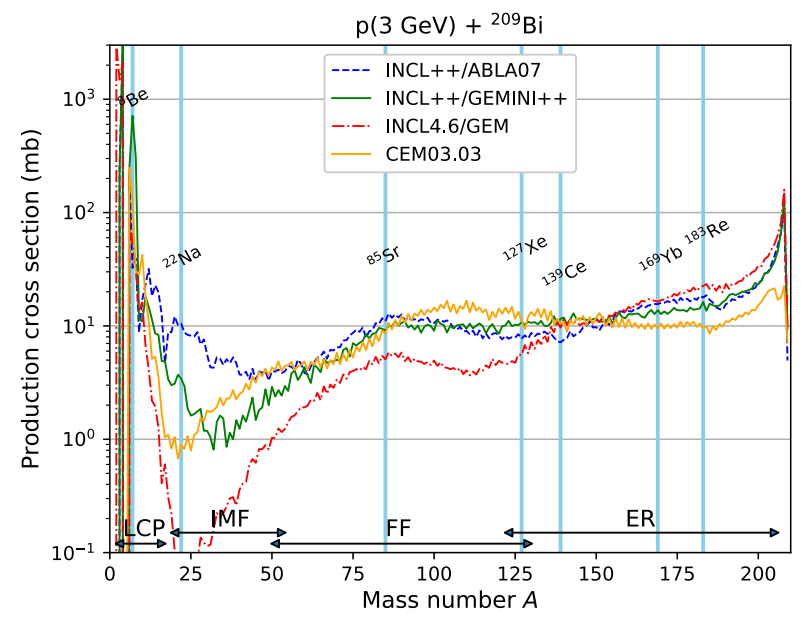

Figure 9. Comparison of mass number distribution between the model calculations for $p(3 \mathrm{GeV})+{ }^{209} \mathrm{Bi}$ reaction. The sky-blue lines indicate mass numbers of the measured isotopes.

tion, together with experimental data measured at RIKEN with the inverse-kinematics technique. Because of the lower incident energy of $185 \mathrm{MeV}$, IMFs are not observed for both the experimental result and the model calculations. Figure 8 compares the mass number distribution of each produced isotopes. INCL++/ABLA07 and INCL++/GEMINI++ show generally good agreement with the experimental data, while underestimation is seen in higher mass numbers of iodine isotopes.

\subsection{Comparison with J-PARC data for ${ }^{209} \mathrm{Bi}$}

In this section, we compare the model calculations with seven pieces of cumulative experimental data measured with the activation technique, as listed in Table 3. Details of this experiment can be found in Matsuda et al. [21]. Figure 9 shows the calculated mass number distributions of the spallation products for the $p(3 \mathrm{GeV})+{ }^{209} \mathrm{Bi}$ reaction. At such a high incident energy, overlaps appear in the mass distributions between ERs, FFs, and IMFs. Figure 10 displays the calculation/experimental $(\mathrm{C} / \mathrm{E})$ values for the cumulative nuclide production cross sections. All of the spallation models estimate the production cross sections for ERs (i.e., ${ }^{139} \mathrm{Ce},{ }^{139} \mathrm{Yb}$, and ${ }^{183} \mathrm{Re}$ ) fairly well; however discrepancies are seen in the FF, IMF, and LCP regions.

Table 3. Experimental production cross sections used for comparison.

\begin{tabular}{rrrr}
\hline \multirow{2}{*}{ nuclide } & half-life & $E_{p}=3 \mathrm{GeV}$ & $E_{p}=400 \mathrm{MeV}$ \\
\hline${ }^{7} \mathrm{Be}$ & $53.22 \mathrm{~d}$ & $10.66 \pm 0.45$ & $0.55 \pm 0.08$ \\
${ }^{22} \mathrm{Na}$ & $2.6018 \mathrm{y}$ & $0.97 \pm 0.09$ & $0.15 \pm 0.02$ \\
${ }^{85} \mathrm{Sr}$ & $64.849 \mathrm{~d}$ & $5.67 \pm 0.31$ & $1.77 \pm 0.10$ \\
${ }^{127} \mathrm{Xe}$ & $36.346 \mathrm{~d}$ & $10.86 \pm 0.45$ & $1.00 \pm 0.04$ \\
${ }^{139} \mathrm{Ce}$ & $137.63 \mathrm{~d}$ & $13.65 \pm 0.50$ & $0.25 \pm 0.01$ \\
${ }^{169} \mathrm{Yb}$ & $32.018 \mathrm{~d}$ & $19.72 \pm 0.74$ & $0.08 \pm 0.02$ \\
${ }^{183} \mathrm{Re}$ & $70.0 \mathrm{~d}$ & $19.75 \pm 1.88$ & $11.37 \pm 1.08$ \\
\hline
\end{tabular}




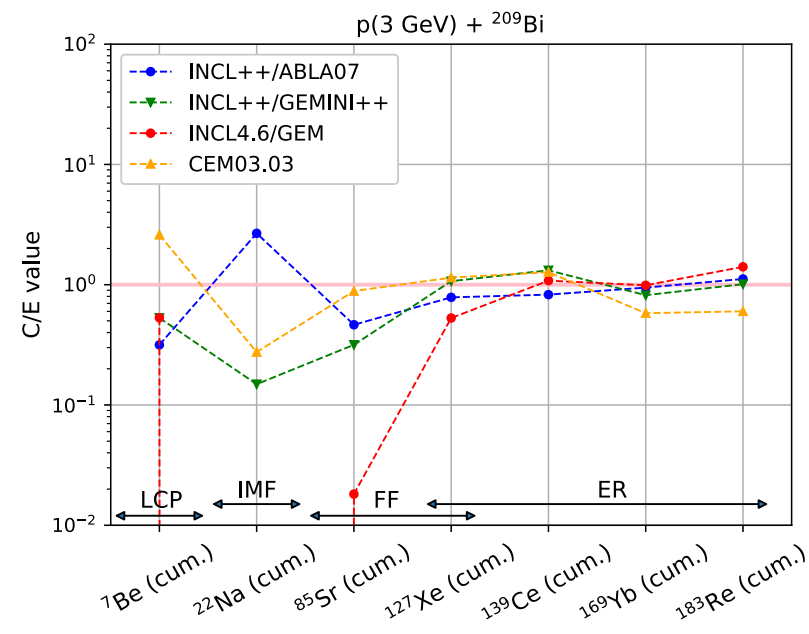

Figure 10. C/E values of the cumulative nuclide production cross sections between the J-PARC experiment and the model calculations for $p(3 \mathrm{GeV})+{ }^{209} \mathrm{Bi}$ reaction.

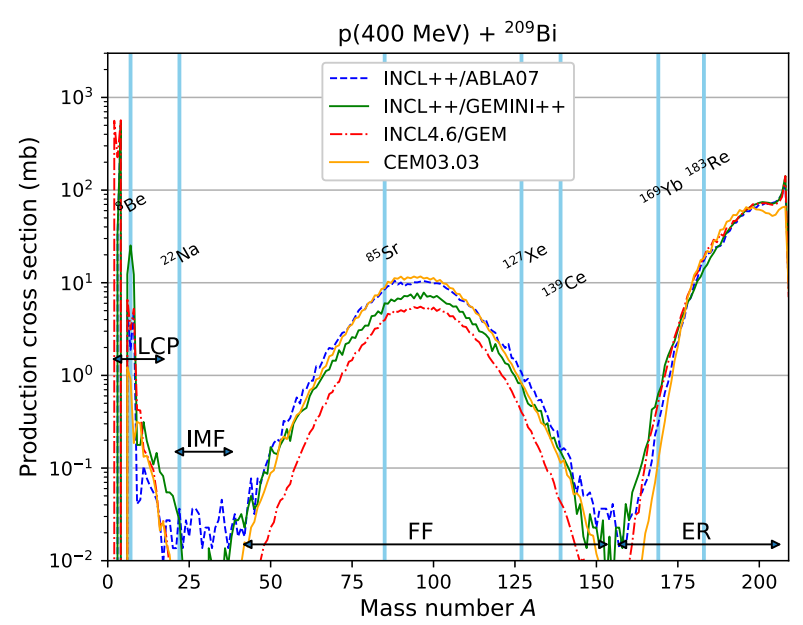

Figure 11. Same as Figure ??, but for $p(400 \mathrm{MeV})+{ }^{209} \mathrm{Bi}$ reaction.

Figure 11 shows the calculated mass number distribution of the spallation products for the $p(400 \mathrm{MeV})+{ }^{209} \mathrm{Bi}$ reaction. Figure 12 is the same as Figure 10, but for the incident energy of $400 \mathrm{MeV}$. All of the spallation models overestimate the ${ }^{169} \mathrm{Y}$ and ${ }^{183}$ Re production cross sections, which lie in the steep slope of the ER region, whereas those of ${ }^{85} \mathrm{Sr},{ }^{127} \mathrm{Xe}$, and ${ }^{139} \mathrm{Ce}$, namely FFs, are underestimated.

\section{Conclusions}

In this study, we have compared the proton-induced nuclide production cross sections between experimental data measured at GSI, RIKEN, and J-PARC and the spallation model calculations by INCL++/ABLA07, INCL++/GEMINI++, INCL4.6/GEM, and CEM03.03. As a result, we have clarified the following issues of each model towards the further improvement of the predictive performance:

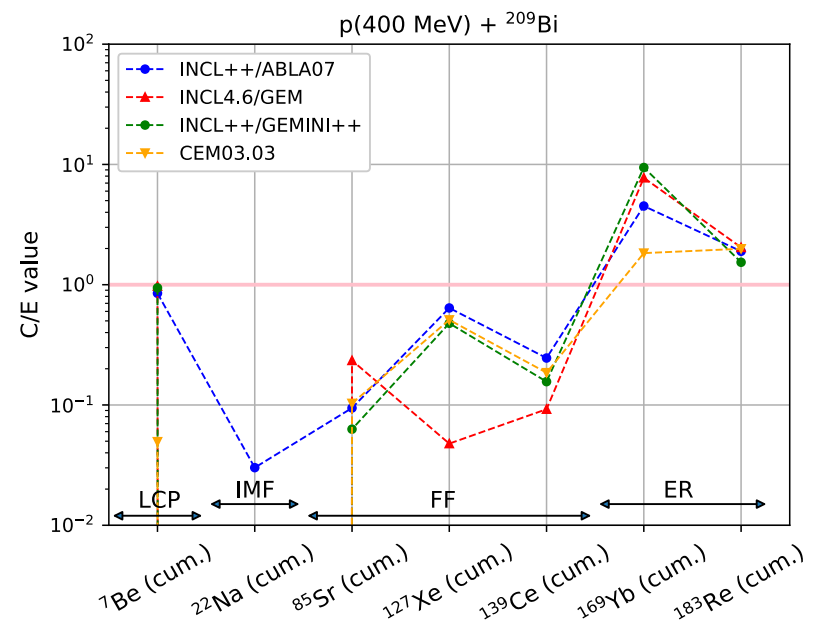

Figure 12. Same as Figure 10, but for $p(400 \mathrm{MeV})+{ }^{209} \mathrm{Bi}$ reaction.

- INCL++/ABLA07 reproduces the GSI and RIKEN experimental data relatively well, which suggests that this model would have better predictive performance of the spallation product yields than other spallation models in calculating the particle transport for ADS and the proton-induced spallation neutron source facilities. However, discrepancies with the J-PARC experimental data were observed. Further improvement of the INCL++/ABLA07 predictive performance requires more detailed validation using both the inverse-kinematicsbased and activation-based experiments.

- INCL++/GEMINI++ estimates ERs as neutron rich for the same $Z$ number, which would be owing to discrepancy in parametrizations between INCL++ and GEMINI++. Although this model reproduces IMF production cross sections for the $p(1 \mathrm{GeV})+{ }^{136} \mathrm{Xe}$ reaction within the experimental uncertainty, underestimation is observed for heavy target nuclei.

- INCL4.6/GEM estimates ERs as neutron rich for the same $Z$ number and underestimates FFs for the ${ }^{208} \mathrm{~Pb}$ and ${ }^{209} \mathrm{Bi}$ targets, which would be owing to discrepancy in parametrizations between the INC and deexcitation models. Furthermore, the GEM model underestimates IMFs. To reproduce the IMF production cross sections, the IMF production mechanism needs to be implemented.

- For the same reason as described above, CEM03.03 estimates ERs as neutron poor for the same $Z$ number. The GEM2 model implemented in CEM03.03 underestimates IMFs. To reproduce the IMF production cross sections, the IMF production mechanism needs to be implemented.

\section{References}

[1] T. Sato, Y. Iwamoto, S. Hashimoto, et al., J. Nucl. Sci. Technol. 55, 684 (2018)

[2] C.J. Werner, J.S. Bull, C.J. Solomon, et al., LA-UR18-20808, LANL, 2018 
[3] S. Agostinelli, J. Allison, K Amako, et al., Nucl. Instr. and Meth. A 506, 250 (2003)

[4] G. Battistoni, T. Boehlen, F. Cerutti, et al., Ann. Nucl. Energy 82, 10 (2015)

[5] J.C. David, D. Filges, F. Gallmeier, et al., Prog. Nucl. Sci. Technol. 2, 942 (2011)

[6] Y. Iwamoto, T. Sato, S. Hashimoto, et al., J. Nucl. Sci. Technol. 54, 617 (2017)

[7] A. Stankovkiy, H. Iwamoto, Y. Çelik, et al., Ann. Nucl. Energy 120, 207 (2018)

[8] S. Leray, D. Mancusi, P. Kaitaniemi, et al., J. Phys.: Conf. Series 420, 012065 (2013)

[9] A. Kelić, M.V. Ricciardi, K.H. Schmidt, INDC(NDS)0530, IAEA, 2008

[10] R.J. Charity, Phys. Rev. C 82, 014610 (2010)

[11] A. Boudard, J. Cugnon, J.C. David, et al., Phys. Rev. C 87, 014606 (2013)

[12] S. Furihata, K. Niita, S. Meigo, et al., JAERI-Data Code 2001-015, JAERI, 1999

[13] S.G. Mashnik, A.J. Sierk, LA-UR-12-01364, LANL, 2012
[14] S.G. Mashnik, K.K. Gudima, A.J. Sierk, Proc. SATIF-6, SLAC USA, OECD/NEA 337, 2004

[15] D. Mancusi, A. Boudard, J. Cugnon, et al., Phys. Rev. C 90, 054602 (2014)

[16] T. Enqvist, W. Wlazło, P. Armbruster, et al., Nucl. Phys. A 686, 481 (2001)

[17] M.V. Ricciardi, P. Armbruster, J. Benlliure, et al., Phys. Rev. C 73, 014607 (2006)

[18] M. Bernas, P. Armbruster, J. Benlliure, et al., Nucl. Phys. A 765, 197 (2006)

[19] P. Napolitani, K.H. Schmidt, L. Tassan-Got, et al., Phys. Rev. C 76, 064609 (2007)

[20] H. Wang, H. Otsu, H. Sakurai, et al., Phys. Lett. B 754, 104 (2016)

[21] H. Matsuda, S. Meigo, H. Iwamoto, et al., Proc. ND2019, Beijing China

[22] N. Otuka, E. Dupont, V. Semkova, et al., Nucl. Data Sheets 120, 272 (2014)

[23] H. Iwamoto, S. Meigo, J. Nucl. Sci. Technol. 56, 160 (2019) 